\title{
Design and Evaluation of an Antenna Applicator for a Microwave Colonoscopy System
}

\author{
Marta Guardiola, Kahina Djafri, Mouloud Challal, Senior Member, IEEE, Miguel A. González \\ Ballester, Gloria Fernandez-Esparrach, Oscar Camara and Jordi Romeu, Fellow, IEEE
}

\begin{abstract}
This paper presents a design of a compact antenna applicator for a microwave colonoscopy system. Although colonoscopy is the most effective method for colorectal cancer detection, it suffers from important visualization restrictions that limit its performance. We recently reported that the contrast between healthy mucosa and cancer was $30-100 \%$ for the relative permittivity and conductivity respectively at $8 \mathrm{GHz}$, and the complex permittivity increased proportionally to the degeneration rate of polyps (cancer precursors). The applicator is designed as a compact cylindrical array of eight antennas attached at the tip of a conventional colonoscope. The design presented here is a proofof-concept applicator composed by one transmitting and one receiving cavity backed $U$-shaped slot antenna elements fed by an L-shaped microstrip line. The antennas are low profile and present a high isolation at $8 \mathrm{GHz}$. The antenna performance is assessed with simulations and experimentally with a phantom composed by different liquids.
\end{abstract}

Index Terms - Cancer detection, colonoscopy, microwave antenna arrays, phantoms.

\section{INTRODUCTION}

$\mathrm{C}$ olorectal cancer (CRC) is the third most commonly diagnosed tumor and the fourth leading cause of cancer death in the world, accounting for about 1.4 million new cases and 774000 deaths in 2015 [1]. The global burden of CRC is expected to increase to $60 \%$ in 2030 [2]. Fortunately, CRC is potentially curable in a $90 \%$ of cases by an early identification and eradication of polyps (cancer precursors) [3]. Colonoscopy is the standard CRC diagnostic approach and is the only method able to remove polyps in the entire colon $[4,5]$. Although colonoscopy screening has significantly increased the survival of CRC patients, still suffers from fundamental limitations [6]. The reduced field of view of the optical camera placed at the tip of the endoscope $\left(<170^{\circ}\right)$, polyp occultation produced by colon angulations and folds, and insufficient bowel preparation, result

Manuscript received March 15, 2018. This work was supported in part by the Catalan Industria del Coneixement program under Grant 2016 PROD 00048 co-financed by FEDER, by Caixaimpulse program under Grant CI1600058, and by the Spanish Inter-Ministerial Commission on Science and Technology (CICYT) under projects TEC201678028-C3-1-P and the Unidad de Excelencia Maria de Maeztu MDM-2016-0600, which is financed by the Agencia Estatal de Investigación, Spain.

M. Guardiola, O. Camara and M.A. Gonzalez-Ballester are with BCN Medtech, Department of Information and Communication Technologies, Universitat Pompeu Fabra, Barcelona, Spain (e-mail: marta.guardiola@upf.edu). in a polyp miss rate of $22 \%$ [7] and a risk of developing cancer after a negative colonoscopy of $8 \%$ [8]. Another limitation of colonoscopy is the subjectivity in the assessment of several characteristics of the lesions such as size, position and type of tissue, which depend on the experience of the endoscopist. As a measure of security, current clinical guidelines [9] recommend removing all lesions found in a colonoscopy study, including small lesions $(<5 \mathrm{~mm})$ that are often benign polyps. A pathologist then analyses all lesions and emits a histology report that is considered the gold standard. This approach is labor- and time- intensive, and significantly increases the medical cost.

In recent years, several devices and technologies have been developed to improve the performance of colonoscopy [10]. High definition (HD) endoscopes and endoscopes with multiple cameras [11] try to increase colon's surface area visualization. Endomicroscopy [11] and virtual chromoendoscopy [12] offer a further layer of improvement by detecting morphological changes in the colonoscopy image that may indicate the presence of a lesion. However, these techniques have a high cost of acquisition, and lack standardized training, diagnostic and staging scores. Mucosal flattening accessories [11] can be incorporated at the endoscope's tip to improve visualization but are only being used in certain indications. Despite these advances, the need to increase detection rates and differentiate malignant and benign tissues during colonoscopy persists. Currently, $12 \%$ of large adenomas (type of polyp prone to degenerate into cancer) of at least $1 \mathrm{~cm}$ are missed even by expert endoscopists using meticulous techniques with the best available equipment. To this aim, advanced computer vision techniques [13] and new imaging modalities exploiting other mechanisms of contrast, different from the optical one, should be investigated. Mads et al. [14] proposed near infrared Raman spectroscopy and we recently investigated the applicability of microwave imaging (MI) $[15,16]$. Table I shows the main features of the different technologies being used to improve

M.A. Gonzalez-Ballester is also with ICREA, Barcelona, Spain

K. Djafri and M. Challal are with Signals and Systems Laboratory, Institute of Electrical and Electronic Engineering, University M'Hamed BOUGARA of Boumerdes, Boumerdes, Algeria. (e-mail: k.djafri@univ-boumerdes.dz).

G. Fernandez-Esparrach is with the Endoscopy Unit, Institut de Malalties Digestives i Metabòliques, IDIBAPS, CIBERehd, Hospital Clínic, University of Barcelona, Barcelona, Spain, (e-mail: mgfernan@clinic.ub.es).

J. Romeu is with the (b) CommSensLab, Signal Theory and Communications Department, Universitat Politecnica de Catalunya, Barcelona, Spain, (e-mail: romeu@ tsc.upc.edu). 
TABLE I

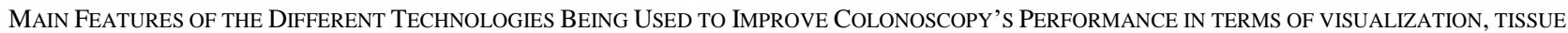
CHARACTERIZATION AND ADR

\begin{tabular}{|c|c|c|c|}
\hline Technology & Colon visualization & Tissue characterization & $\mathrm{ADR} \%$ increase $^{\mathrm{a}}$ \\
\hline HD colonoscopy & $\begin{array}{l}\text { Optical sensor captures more images per } \\
\text { second, } \leq 170^{\circ} \mathrm{FOV}^{\mathrm{b}}\end{array}$ & More resolution & $8.2 \%(\mathrm{p}=0.002)$ \\
\hline $\begin{array}{l}\text { Virtual chromo- } \\
\text { endoscopy }\end{array}$ & $\leq 170^{\circ} \mathrm{FOV}$ & $\begin{array}{l}\text { Narrow optical spectrum to increase } \\
\text { the vascular contrast of the mucosa }\end{array}$ & No significant differences \\
\hline $\begin{array}{l}\text { Cap-assisted colonoscopy } \\
\text { (Endocuff) }\end{array}$ & $\begin{array}{l}\text { Disposable cuff attached at the distal tip of the } \\
\text { colonoscope to flatten colon folds }\end{array}$ & No & $\begin{array}{l}4.8 \%(\mathrm{p}=0.002) \text { in bowel cancer } \\
\text { screening population }\end{array}$ \\
\hline $\begin{array}{l}\text { Full spectrum endoscopy } \\
\text { (FUSE, Aer-O-Scope) }\end{array}$ & Multi-lens $\left(330^{\circ}, 360^{\circ} \mathrm{FOV}\right)$ & No & Inconclusive evidence \\
\hline
\end{tabular}

${ }^{a}$ ADR (Adenoma Detection Rate) is most important indicator of quality in colonoscopy and measures the proportion of colonoscopies in which at least one adenoma is found. This increase is referenced to conventional colonoscopy. ${ }^{b} \mathrm{FOV}$ is the Field Of View of the image acquisition system.

conventional colonoscopy.

MI involves illuminating a body under test with nonionizing and low power microwave signals [17]. Depending on the contrast in complex permittivities of the body, different amount of scattering is produced. This scattering is then collected by the receiving antennas and transformed into images of the body's permittivity profile using MI algorithms. We recently demonstrated that the complex permittivity correlates with the degeneration grade of colon polyps $[15,16]$; the more similar to cancer, the higher the relative permittivity and conductivity. The maximum contrast between healthy mucosa and cancer was obtained between 5 and $8 \mathrm{GHz}$ and was $30-100 \%$ for the relative permittivity and conductivity, respectively. Accordingly, MI offers a contrast mechanism to improve the detection of subtle/flat lesions and to fill the gap between endoscopists with different levels of experience. MI is able to form $360^{\circ}$ images (conventional colonoscopy is limited to $170^{\circ}$ ) with a fair tradeoff between resolution and tissue penetration, that may help to detect the polyps hidden by colon angulations and folds. Therefore, MI has the potential to both reduce the visualization problems of conventional colonoscopy and provide differential diagnosis of benign and malignant polyps during colorectal examinations. MI systems generally consist of a microwave signal transmitter and receiver, such as a vector network analyzer, and an applicator composed by an antenna array and a switching system to select the active antenna elements in each acquisition. Such a hardware for MI can be produced at a fraction of the price of other medical diagnostic equipment, resulting in a low-cost system [18]. As MI does not require contact with the body, it may be easily integrated to colonoscopy's tubes without changing the current clinical practice.

Our aim is to integrate MI with conventional colonoscopy in a multimodal approach. As far as we know, this is the first time that MI is proposed for endoscopic applications. Microwaves have been established as a good solution for treatment of cancer (hyperthermia, and ablation) [19], vital signs monitoring (respiration and heart beat detection) [20], and there is ongoing research studying MI for image-based diagnosis in an extensive number of applications, e.g., bone imaging [21], heart imaging [22], etc., being breast cancer [23] and brain stroke detection [24] the most researched and advanced topics.

In this paper we design and test the antennas for a future MI system for endoscopic applications and we validate its capacity to detect malignant polyps with a simple phantom. The remainder of this paper is organized as follows. Section II describes the challenges and requirements for a MI system for colonoscopy. In section III we describe the antenna applicator, including the design and simulation of the individual antenna element and the planar antenna array. Section IV, presents the imaging method and shows the experimental imaging results of a phantom composed by different liquids. Finally, section V concludes the paper.

\section{SySTEM REQUIREMENTS AND CHALLENGES}

A colonoscope is a thin flexible tubular instrument used to examine the interior of a hollow organ or cavity in the body equipped with a light delivery system, a lens and a camera at its tip. The colon is a tubular organ located in the last part of the gastrointestinal tract of $1.5 \mathrm{~m}$ in length and between $5-10 \mathrm{~cm}$ in diameter with a segmented appearance (i.e. colon folds). Colon polyps are superficial tumors that develop from healthy mucosa and present several shapes, i.e., protruded, slightly elevated, flat, depressed, etc., the first two being the most common. During colonoscopy, the colon is generally inflated with air or $\mathrm{CO}_{2}$ and previously cleaned with a diet and medication to facilitate the camera vision.

The antenna applicator is designed to avoid interfering the optical visualization system at the tip of the endoscope while being safe for the patient and maneuverable for the endoscopist. To meet these requirements, the applicator is designed as a cylindrical compact array attached at the tip of a conventional colonoscope, see Fig. 1. This design is widely used in

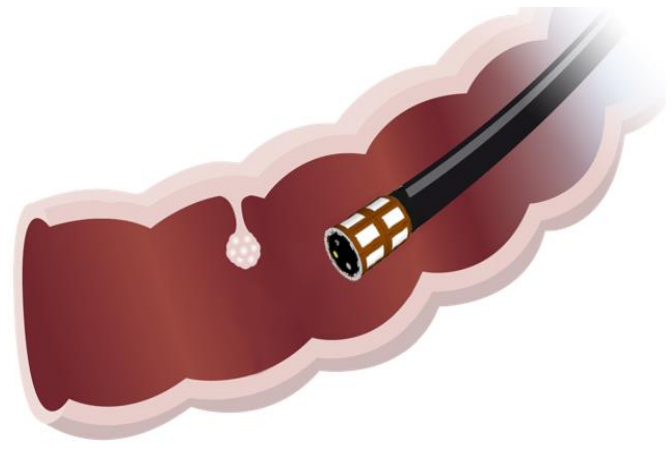

Fig. 1. Diagram of the proposed MI system for colonoscopy including the antenna applicator attached at the tip of a conventional colonoscope. 
colonoscopy accessories [10] and accepted by doctors.

One of the main challenges in endoscopic applications is the size restriction. For this design, we took as a reference a videocolonoscope from Olympus with an insertion tube diameter of $13.2 \mathrm{~mm}$. According to clinicians' opinions the maximum thickness of the applicator to avoid patient discomfort is $5 \mathrm{~mm}$. The length is also limited to avoid restricting the colonoscope bending capacity. Large number of antenna elements is generally desired as it provides more angular resolution and facilitates the solution of the inverse problem. When the antennas are very close together, mutual coupling increases introducing error in the measured scattered field unless we take measures to reduce it. To meet the size limitation of the intended application, we have selected a compact antenna design, limited the number of elements in the array and minimized the separation between them. The diameter of the cylindrical array is set to $23.2 \mathrm{~mm}$ and its length to $30 \mathrm{~mm}$.

In general, very high dynamic range systems are needed to be able to recognize a weakly scattering target (e.g. a tumor) within a high clutter environment. In endoscopic applications penetration is not required as polyps are superficial lesions, thus relaxing the previous requirement.

The choice of the working frequency band is one of the key aspects of a MI system. The well-known tradeoff between resolution and penetration results in an optimal working frequency range around a few $\mathrm{GHz}$ for most medical applications. As said, in this case penetration is not needed, however higher frequencies are more sensitive to noise. Robustness is one of the main requirements for using a MI system in a clinical environment that is especially rich in electromagnetic interferences and noise, which can lead to errors in the measured data. For this application, the frequency range 5-8 GHz gives the highest contrast among healthy colon, different types of polyps and cancer $[15,16]$. Furthermore, realtime, computationally-efficient and accurate imaging algorithms are required.

Other challenges linked to endoscopic applications are the movement of the endoscope as the it travels through the colon and the movement of the colon itself. The unknown position of the antenna array with respect to the colon walls and the presence of colon folds and angulations, may produce reflections that eventually may mask the polyp's response.

\section{DESIGN OF THE ANTENNA APPLICATOR}

The applicator array consists of sixteen identical cavitybacked U-slot elements fed by sixteen L-shape microstrip lines. The elements are arranged in two rows of eight elements each. One row is used for transmission and the other for reception. The elements are wrapped around a conducting cylinder with sixteen recessed cavities. As illustrated in Fig. 2, antenna elements on the same row are separated by metallic walls of a width of $1 \mathrm{~mm}$ while transmission and reception rows are separated by a metallic disk of a thickness of $4 \mathrm{~mm}$ and a diameter of $23.2 \mathrm{~mm}$. For feeding convenience, each antenna element is backed by an open cavity such that the cavity comprises only three metallic walls. Accordingly, no metallic

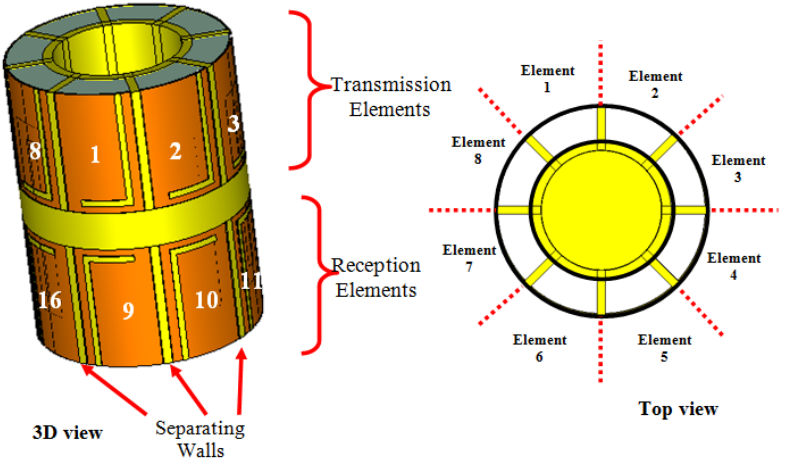

Fig. 2. Geometry of the cylindrical U-slot antenna array.

wall is present in the feeding side of each antenna element.

\section{A. Antenna Element Design}

The antenna element used in the applicator is a cavitybacked U-slot fed by an L-shape microstrip line. This design is selected to meet the reduced size requirements of the application and enhance the isolation between array elements. The slot is printed on RT/Duroid 5880 substrate having a relative permittivity of 2.2 and a thickness of $0.127 \mathrm{~mm}$. The substrate is very flexible and thus can be easily conformed onto a cylindrical surface. An L-shape microstrip line printed on the back side of the substrate is used to excite the slot. The cavity is filled with a lossy foam with relative permittivity of 2.1 as illustrated in Fig. 3 (a). The size of the cavity is $13 \times 8.16 \mathrm{~mm}^{2}$, which is shown by the external dashed rectangle in Fig. 3 (b), and the cavity depth is $4 \mathrm{~mm}$. The geometrical parameters of the backed-cavity U-slot microstrip-fed antenna are shown in Table II.

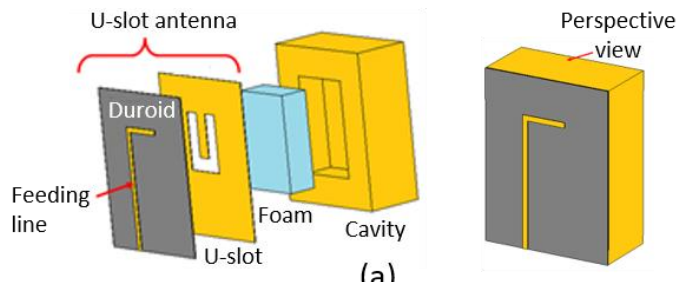

(a)

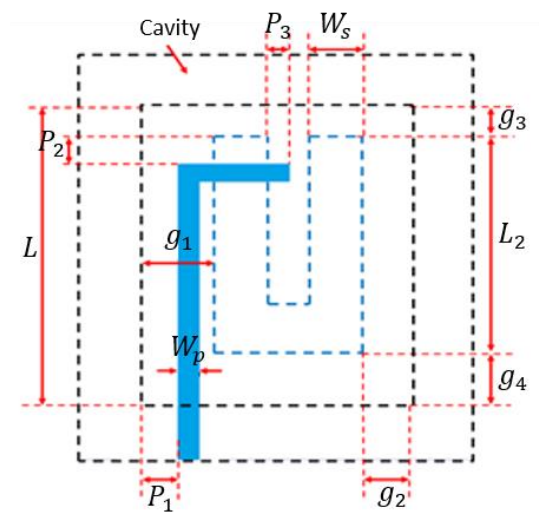

(b)

Fig. 3. Geometry of the cavity-backed U-slot antenna element. (a) Cavity with the U-slot antenna, (b) Top view. 
TABLE II

PROPOSED ANTENNA ELEMENT DiMENSIONS.

\begin{tabular}{cc|cc}
\hline \hline Parameter & Value $(\mathrm{mm})$ & Parameter & Value $(\mathrm{mm})$ \\
\hline$L$ & 13 & $P_{1}$ & 1 \\
$g_{1}$ & 1 & $P_{2}$ & 0 \\
$g_{2}$ & 1.84 & $P_{3}$ & 3.72 \\
$g_{3}$ & 0.75 & $W_{s}$ & 1.8 \\
$g_{4}$ & 4.55 & $W_{p}$ & 0.65 \\
$L_{2}$ & 7.7 & & \\
\hline \hline
\end{tabular}

The cavity-backed U-slot antenna is designed to operate inside the human colon. Consequently, the presence of colon tissue must be considered in the analysis. The simulated reflection coefficients for different distances $\mathrm{D}$ between the antenna and the colon tissue are presented in Fig. 4. The colon tissue is modeled as a box of $52 \times 52 \times 10 \mathrm{~mm}^{3}$. For the material, we assigned the dispersive dielectric properties of colon mucosa reported in [16]. It can be observed that the antenna input impedance matching is affected by the presence of the biological tissue. The minimum level of the input reflection coefficient is less than $-10 \mathrm{~dB}$ for all simulated distances. Furthermore, the antenna resonant frequency decreases slightly as the distance between the antenna and the colon tissue increases.

\section{B. Array of two Antenna Elements}

Since the antenna array will be used in switched-beam mode, the isolation between antenna elements is crucial. Accordingly, two configurations of two adjacent antennas were investigated as shown in Fig. 5. The first configuration consists of two adjacent antenna elements, one used for transmission and the other for reception, namely transmission-reception configuration. In the second configuration two adjacent antenna elements are located in the same row dedicated either for transmission or for reception, namely side by side configuration.

The simulated return loss (S11) and the mutual coupling (S21) of the transmission-reception configuration with and without colon tissue for different distances from colon tissue is illustrated in Fig. 6. It is clear from the figures that these parameters are affected by the presence of the colon tissue. As

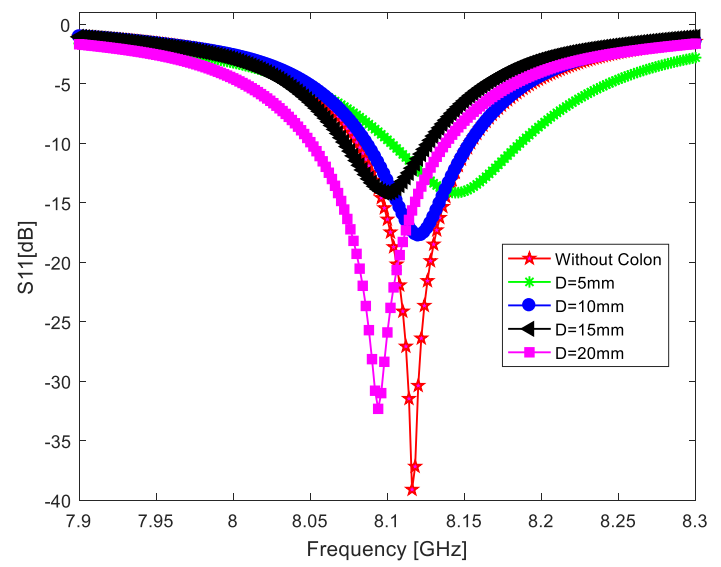

Fig. 4. Simulated reflection coefficient of one antenna element for different distances $\mathrm{D}$ between the antenna and the colon tissue.
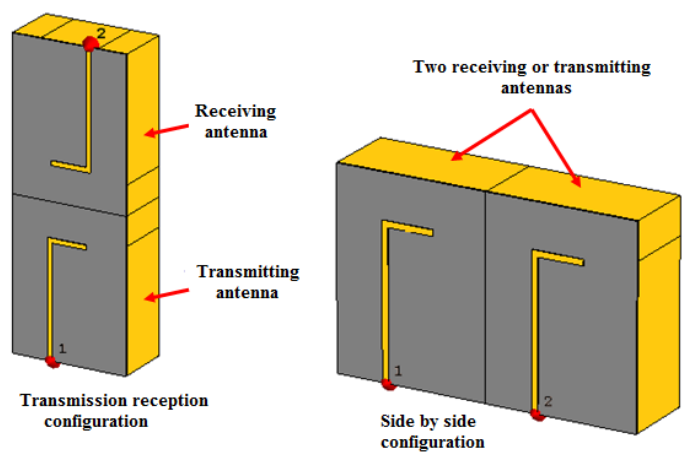

Fig. 5. Studied antenna configurations: transmission-reception configuration (left), and side by side configuration (right).

the distance $\mathrm{D}$ between the antennas and the colon wall increases, the input impedance matching is improved. Also, the antenna resonant frequency decreases slightly as D increases. The antenna return loss is less than $-15 \mathrm{~dB}$ for all simulated values of $\mathrm{D}$, and the coupling between two antennas is always lower than $-12 \mathrm{~dB}$, denoting an acceptable isolation between elements. As expected, the isolation improves as the distance between the antenna and the colon wall increases.

For the side by side configuration, the simulated return loss and the mutual coupling with and without colon tissue for different distances D are presented in Fig. 7. Similarly to the previous configuration, the antenna matching and the resonant frequency are affected by the presence of the colon tissue. In this case the antenna reflection coefficient is lower than $-10 \mathrm{~dB}$ and the isolation is lower than $-15 \mathrm{~dB}$ for all assumed values of D.
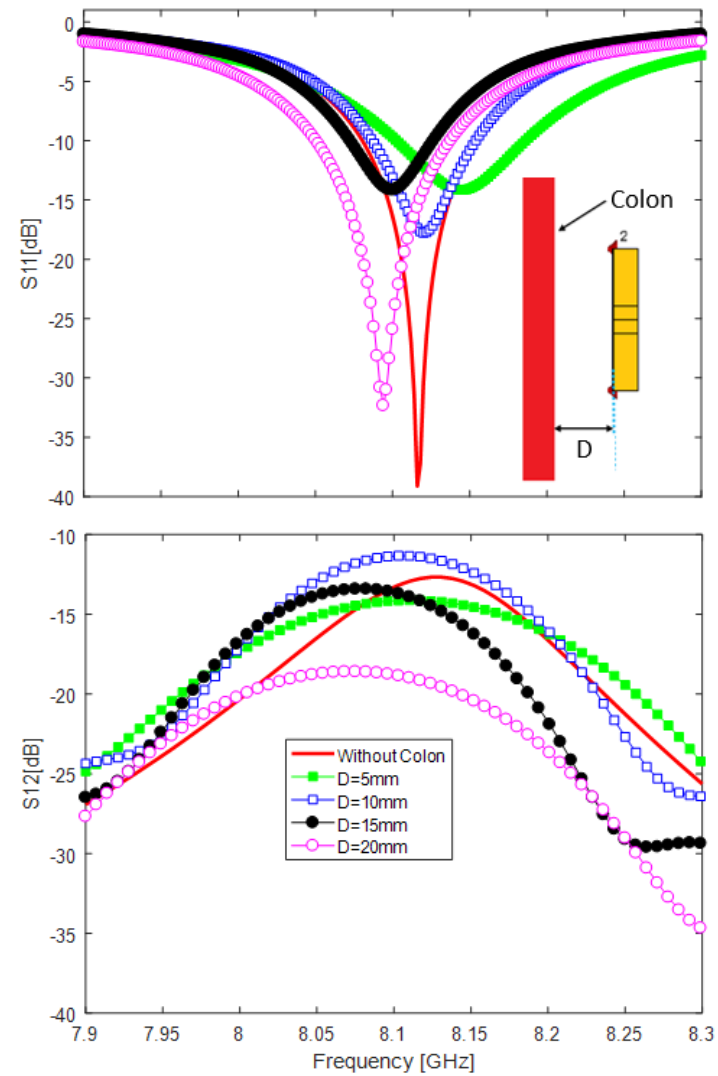

Fig. 6. Simulated return loss (top), and mutual coupling (bottom) for different values of $\mathrm{D}$ for transmission-reception configuration. 

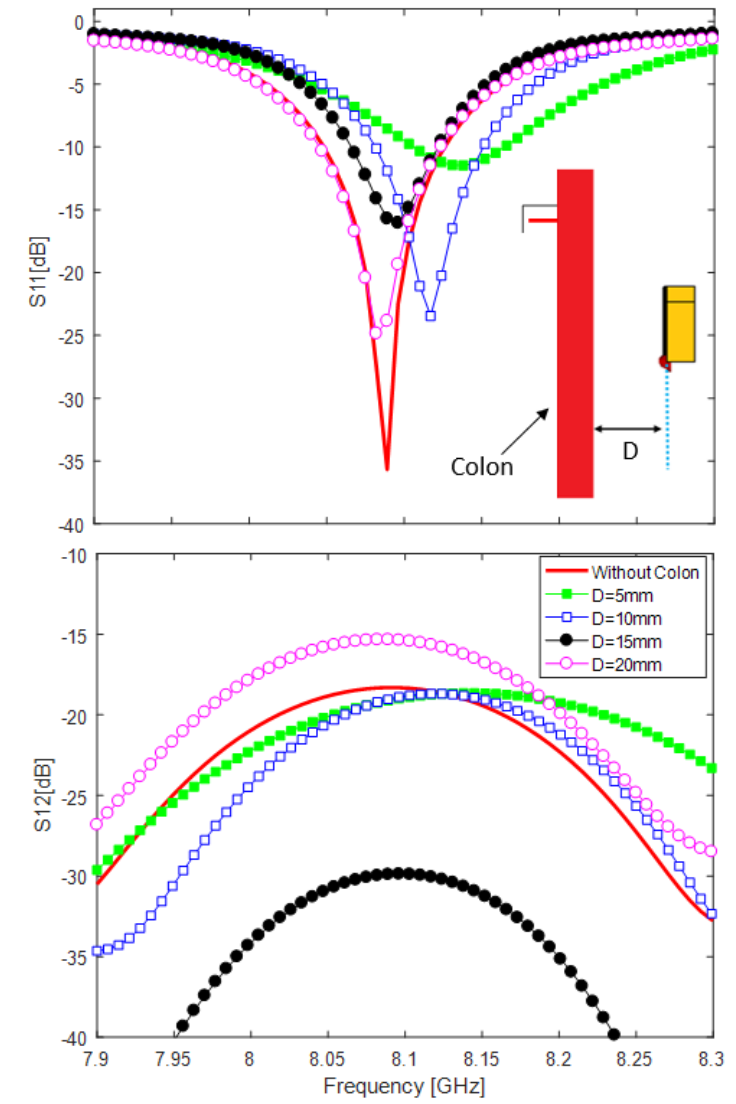

Fig. 7. Simulated return loss (top), and mutual coupling (bottom) for different values of $\mathrm{D}$ for side by side configuration.

\section{Fabrication and Measurement of the Antennas}

Two identical antennas are fabricated, one working as a transmitter and the other as a receiver and assembled on a metal surface (referred as the applicator in the rest of the paper). The antennas are connected to a two-port commercial Agilent performance network analyzer (PNA) with flexible coaxial cables to measure the S-parameters. Fig. 8 shows a picture of the fabricated antennas. Fig. 9 shows the measured and simulated return loss for one antenna element without colon tissue. It can be observed a very good agreement between the measured and simulated return loss.

The measured and simulated return loss and isolation for the side by side configuration are illustrated in Fig. 10. It can be observed that the simulated and measured results exhibit a good agreement. The small difference between them is possibly due to fabrication errors. The measured isolation is lower than -30 $\mathrm{dB}$ while the simulated one is lower than $-17 \mathrm{~dB}$. This difference may be produced by the presence of two metal walls

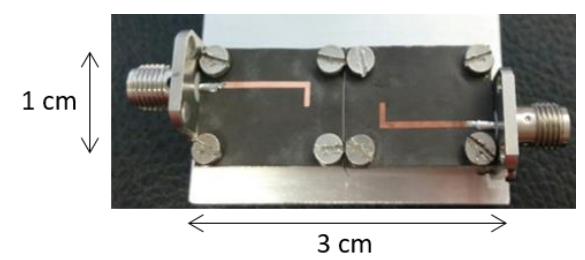

Fig. 8. Picture of the fabricated antennas in transmission-reception configuration.

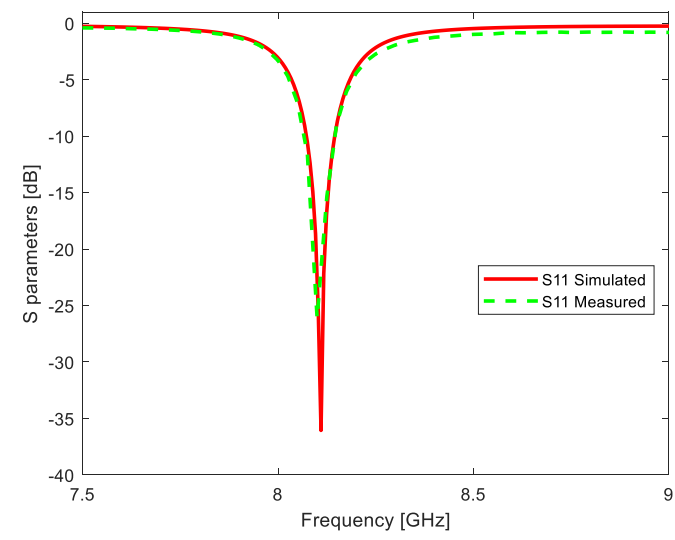

Fig. 9. Simulated and measured return loss for one antenna element without colon tissue.

between the transmitting and receiving antennas in the fabricated applicator, whereas only one wall existed in the simulations. The measured isolation is lower than $-25 \mathrm{~dB}$ for the transmission-reception configuration.

\section{EXPERIMENTAL FUNCTIONAL EVALUATION}

The experimental evaluation is performed with a virtual planar antenna array composed by two antenna elements in transmission-reception configuration. A flat applicator is better suited to phantoms and ex vivo samples with which the system will be validated in the future. The applicator is attached on a two-axis motorized linear positioner to obtain raster scans at the surface of the sample under test as shows Fig. 11. The scanned area is sampled in $N_{y}=8$ steps separated $1 \mathrm{~cm}$ along y-axis to
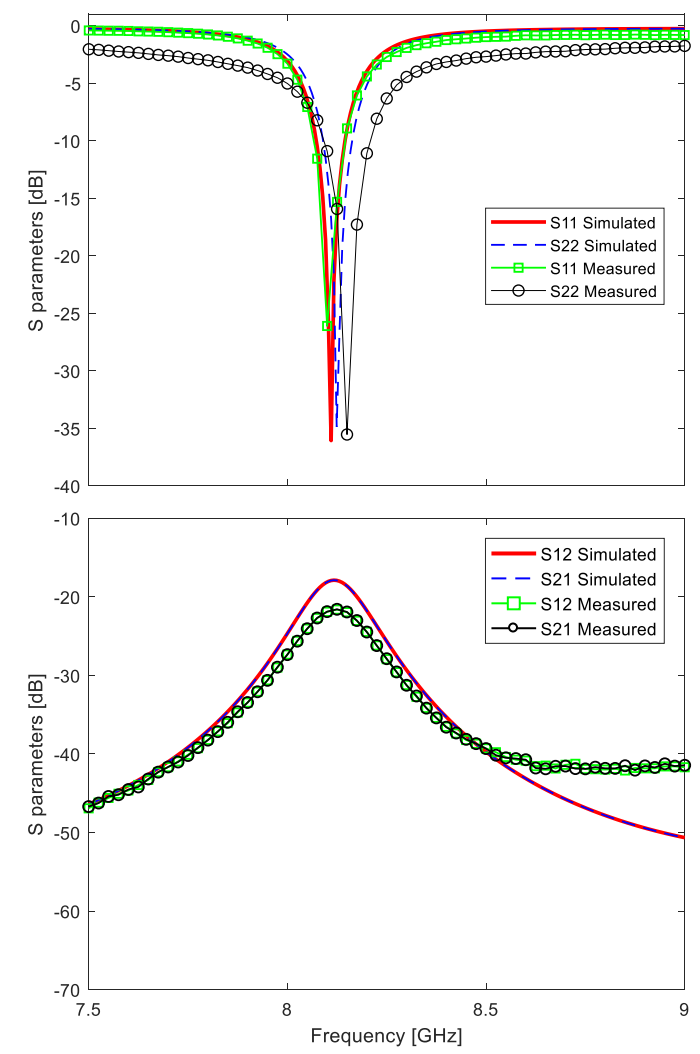

Fig. 10. Simulated and measured return loss (top), and isolation (bottom) for side by side configuration without colon tissue. 
synthesize a virtual array, and in $N_{x}=8$ steps separated $1 \mathrm{~cm}$ along $\mathrm{x}$-axis.

The phantom is composed by methanol and deionized water mixtures mimicking healthy colon tissue and a malignant polyp respectively. Fig. 12 shows the dielectric properties of the mixtures measured with an open-ended coaxial probe. The colon mimicking liquid was poured in a $130 \times 130 \times 40 \mathrm{~mm}^{3}$ plastic container. For the polyp, we used two cylindrical plastic containers of $10 \mathrm{~mm}$ and $20 \mathrm{~mm}$ in diameter respectively. The phantom was placed below the antennas. The distance between the antennas and the phantom's surface is $30 \mathrm{~mm}$.

\section{A. Reconstruction Algorithm}

A cross-sectional image of the colon phantom is obtained for each measurement plane. For each plane, a set of $N_{y}=8$ transmitters and $N_{y}=8$ receivers are used to scan the colon area where the reconstruction algorithm is applied. First, a measurement matrix is obtained as follows: for every frequency and transmitting position, the scattered field is measured over one receiver position in a bistatic approach, obtaining a $1 \times N_{f}$ measurement vector, where $N_{f}$ is the number of frequencies. Then, the Y-positioner moves one step and the receiver performs another measurement. The procedure is repeated for the $N_{y}$ pairs of transmitting and receiving elements, obtaining a $N_{y} \times N_{f}$ matrix. Next, the X-positioner moves one step and the procedure is repeated. The measurement is completed after repeating the previous process for the $N_{x}=8$ movements of the $\mathrm{X}$-positioner (called planes from now on) obtaining a $N_{x} \times N_{y} \times N_{f}$ matrix, $E_{i}^{S}$, where $i=1 . . N_{y}$ indexes the planes.

The received fields are masked by substantial amount of reflections from the background, cables, the air-phantom interface, among others. To extract the useful scattered signals from the target (e.g. the water bubble), a calibration is

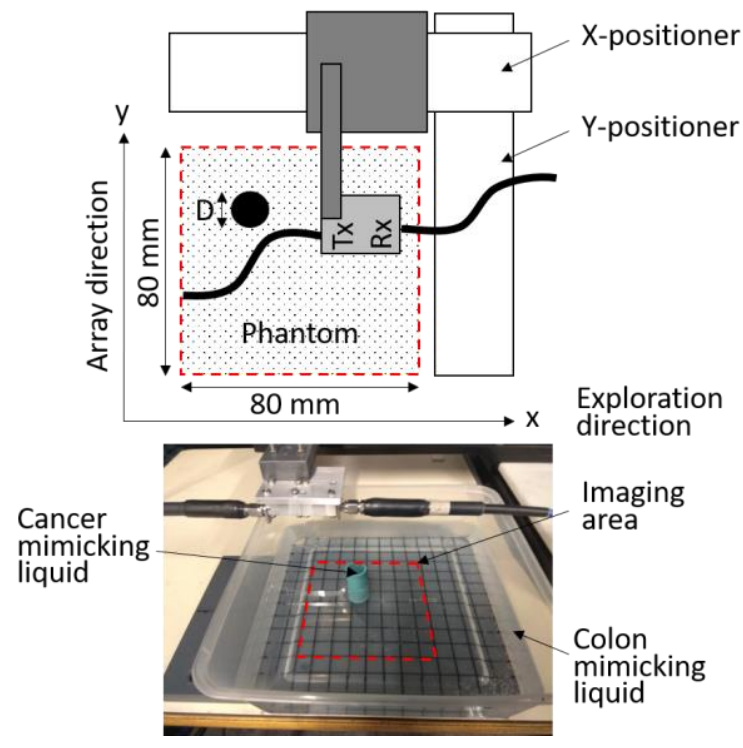

Fig. 11. Virtual antenna array system consisting of a pair of transmitting and receiving antennas mounted on a two-axis positioner. The phantom is composed of two methanol and deionized water mixtures poured in plastic containers that model colon tissue and a polyp, respectively (top). Picture of the antennas over the phantom (bottom). The red rectangle shows the imaging area.

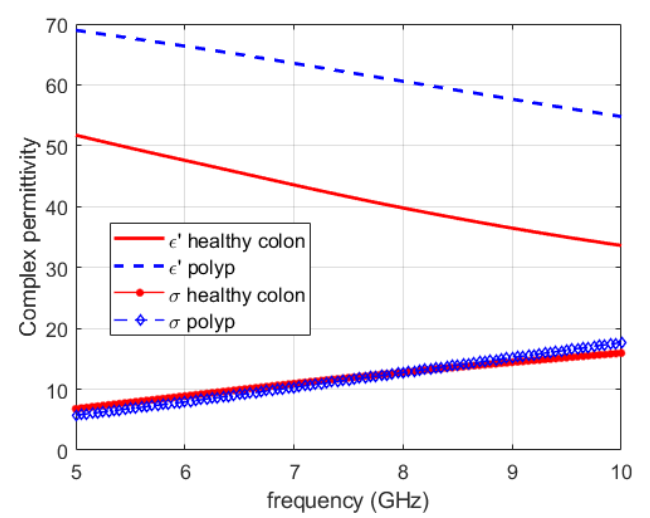

Fig. 12. Measured relative permittivity and conductivity of healthy colon mimicking liquid mixture and malignant polyp liquid mimicking mixture.

performed. We first measure the electric fields without phantom (empty), and we compute the scattered field, $E^{s_{1}}$, subtracting the empty measurement from the scattered field obtained with the phantom.

For each plane, a cross-sectional image is obtained as shows Fig. 13. A confocal or radar-based method [25] namely Bifocusing algorithm [26] is used. The imaging area is divided into cells defined by their center point vector $\vec{r}$. The reconstruction algorithm formed every image point of the local frequency averaged electric contrast of the object, defined as $c(x, y)=\left(\epsilon^{\prime}(x, y)-\epsilon_{b}^{\prime}\right) / \epsilon_{b}^{\prime}$, using the following equation:

$$
\tilde{c}_{i}(\vec{r})=\sum_{m=1}^{N_{f}}\left|\sum_{n=1}^{N_{x}} \frac{E_{i}^{S}\left(\vec{r}_{T_{n}}, f_{m}, \vec{r}_{R_{n}}\right)}{k_{m}^{2} H_{0}^{2}\left(k_{m}\left|\vec{r}_{T_{n}}-\vec{r}\right|\right) H_{0}^{2}\left(k_{m}\left|\vec{r}_{R_{n}}-\vec{r}\right|\right)}\right|
$$

where $\epsilon_{b}^{\prime}$ is the relative permittivity of the background medium, $\epsilon^{\prime}(x, y)$ is the relative permittivity of each phantom's point, and $k=2 \pi f \sqrt{\mu_{b} \epsilon_{b}}$ is the wavenumber. $H_{0}^{2}$ is the second kind zeroorder Hankel function that is the Green's function for a $2 \mathrm{D}$ geometry, and $\vec{r}_{T_{n}}$ and $\vec{r}_{R_{n}}$ are the position vectors of the $\mathrm{n}^{\text {th }}$ transmitting and receiving antennas, respectively. In (1) the received scattered fields resulting from all the antenna pairs are numerically weighted by a focusing operator focused on a unique image point defined by vector $\vec{r}$. This numerical focusing operator restores the amplitude and phase changes suffered by a wave in its way from the transmitting antenna passing through every image point and ending to the receiving antenna. For each image point, the coherent summation of all

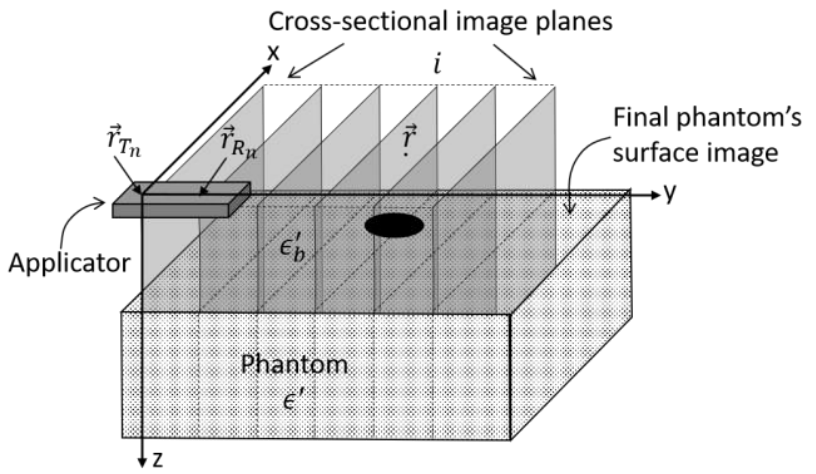

Fig. 13. A final continuous color image of the phantom's surface was obtained by selecting the proper row of each cross-sectional image. 


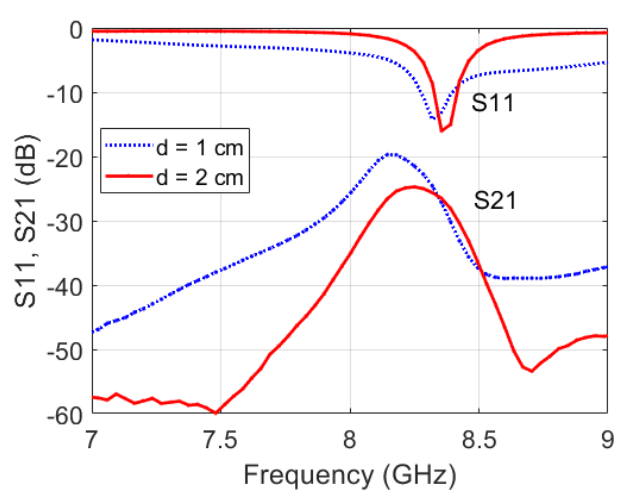

Fig. 14. S-parameters between two antennas separated $\mathrm{d}=1 \mathrm{~cm}$ and $\mathrm{d}=2 \mathrm{~cm}$ from the surface of a water container.

antenna contributions results in a large intensity value if the actual point is the origin of the scattering. If not, the summation results in a small value and this contribution can be considered as noise. A final continuous color image of the phantom's surface is then obtained by selecting the proper row of each cross-sectional image, as shows Fig. 12. High intensity (normalized, thus highest intensity is 1) of colors indicates the positions of the strongest scatterers (e.g. the polyp) on the phantom.

\section{B. Experimental Results}

To experimentally assess the performance of the antennas in presence of a high permittivity material (e.g. the colon), we first measure the S-parameters between the transmitting and receiving antennas in presence of deionized water. To this aim, the applicator is placed over a water container and we vary the distance $d$ between the antenna and the water's surface. In Fig.
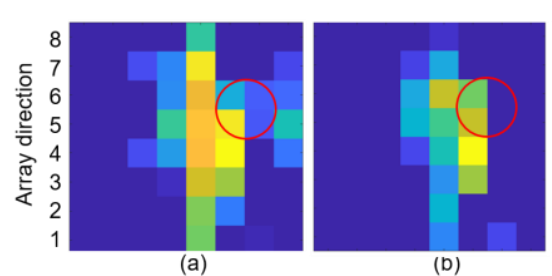

(D)

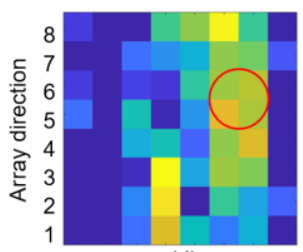

(d)

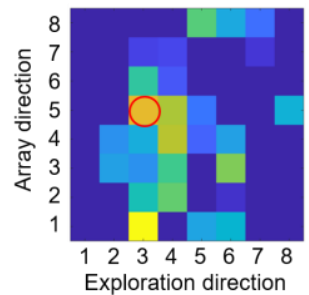

(g)

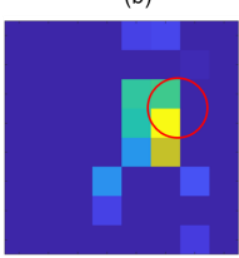

(e)

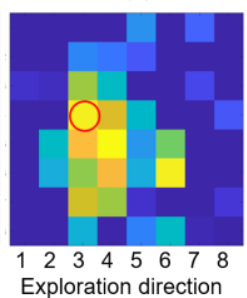

(h)

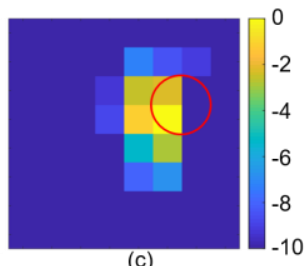

(c)

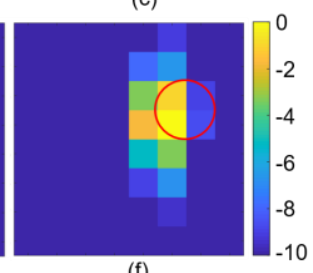

(f)

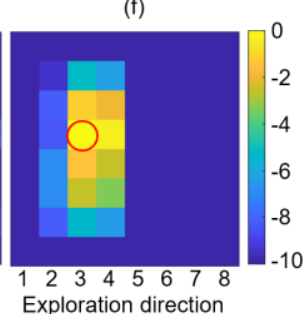

(i)
Fig. 15. Normalized scattered field measured at the surface of the phantom for different frequencies and polyp configurations. Flat polyp of $20 \mathrm{~mm}$ : (a) 6 $\mathrm{GHz}$, (b) $7 \mathrm{GHz}$, (c) $8 \mathrm{GHz}$. Protruded polyp of $20 \mathrm{~mm}$ : (d) $6 \mathrm{GHz}$, (e) $7 \mathrm{GHz}$, (f) $8 \mathrm{GHz}$. Protruded polyp of $10 \mathrm{~mm}:(\mathrm{g}) 6 \mathrm{GHz}$, (h) $7 \mathrm{GHz}$, and (i) $8 \mathrm{GHz}$. The red circle indicates the real position of the polyp.
14 we can observe a clear shift of the peak towards lower frequencies in both S11 and S21 when the distance varies from $2 \mathrm{~cm}$ to $1 \mathrm{~cm}$. This result agrees with simulations and suggests that the antennas are quite sensitive to geometric changes in the sample under test.

The phantom is then used to assess the capacity of the antennas to identify different colon lesions. Fig. 15 shows the normalized measured scattered field in $\mathrm{dB}$ scale at the surface of the phantom for three different phantom configurations using the calibration method explained before. For each configuration, three frequencies are measured $(6,7$ and $8 \mathrm{GHz})$, that are within the frequency band reporting more dielectric contrast between different colon tissues [16]. The first row corresponds to a colon tissue with a circular flat polyp of $20 \mathrm{~mm}$ in diameter. A flat polyp is very difficult to detect with optical colonoscopy because it is visually confused with the neighboring tissue. This scenario has been modeled pouring the same level of liquid in the containers that simulate the colon and the polyp respectively. The second and the third row correspond to a colon tissue with a protruded polyp of $20 \mathrm{~mm}$ and $10 \mathrm{~mm}$ respectively at different positions. In this case, we have poured less amount of liquid into the container that models the colon, specifically one centimeter less. Therefore, the polyp protrudes $10 \mathrm{~mm}$ from the colon surface. Fig. 15 presents stronger field values over the polyp, marked with a red circumference, while the background presents lower values. At 7 and $8 \mathrm{GHz}$ the position of the polyp can be inferred directly from the measured field, while at $6 \mathrm{GHz}$ the image presents more artifacts. As expected, the best results are obtained at $8 \mathrm{GHz}$, in which the antennas are well matched.

Fig. 16 shows the reconstructed images obtained by applying

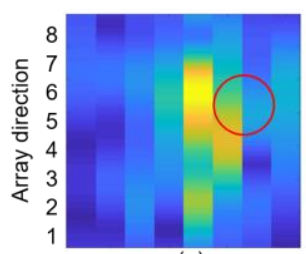

(a)

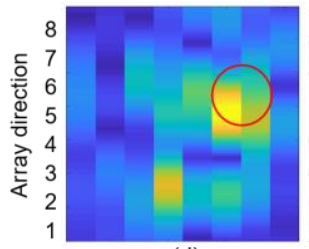

(d)

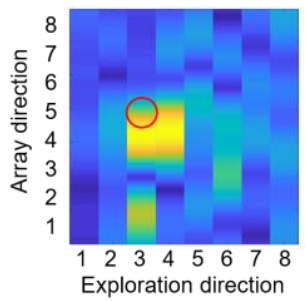

(g)

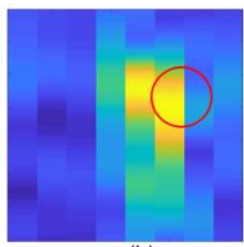

(b)

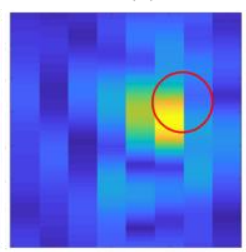

(e)

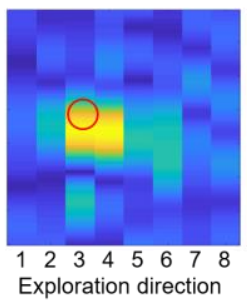

(h)

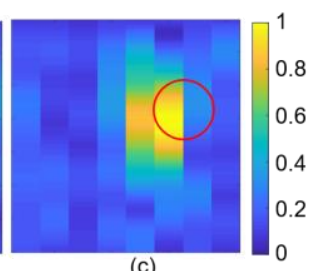

(c)

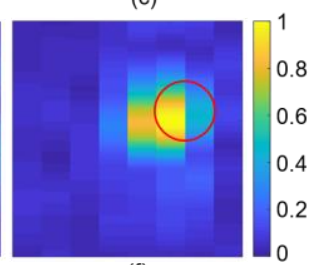

(f)

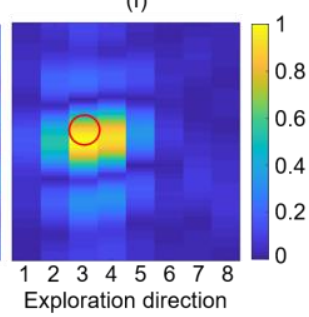

(i)
Fig. 16. Reconstructed images of the normalized electric contrast at the phantom's surface for different frequencies and polyp configurations. Flat polyp of $20 \mathrm{~mm}$ : (a) $6 \mathrm{GHz}$, (b) $7 \mathrm{GHz}$, (c) $8 \mathrm{GHz}$. Protruded polyp of $20 \mathrm{~mm}$ : (d) $6 \mathrm{GHz}$, (e) $7 \mathrm{GHz}$, (f) $8 \mathrm{GHz}$. Protruded polyp of $10 \mathrm{~mm}:$ (g) $6 \mathrm{GHz}$, (h) 7 $\mathrm{GHz}$, and (i) $8 \mathrm{GHz}$. The red circle indicates the real position of the polyp. 
TABLE III

MEtrics For the Reconstruction of the Phantom: SCR, FWHM, PA ARe Signal to Clutter Ratio, Full Width Half Maximum and Position ACCURACY, RESPECTIVELY

\begin{tabular}{|c|c|c|c|c|c|c|c|c|c|}
\hline & \multicolumn{3}{|c|}{ Flat } & \multicolumn{3}{|c|}{$\begin{array}{c}\text { Protruded } \\
\mathrm{D}=20 \mathrm{~mm}\end{array}$} & \multicolumn{3}{|c|}{$\begin{array}{c}\text { Protruded } \\
\mathrm{D}=10 \mathrm{~mm}\end{array}$} \\
\hline & $6 \mathrm{GHz}$ & $7 \mathrm{GHz}$ & $8 \mathrm{GHz}$ & $6 \mathrm{GHz}$ & $7 \mathrm{GHz}$ & $8 \mathrm{GHz}$ & $6 \mathrm{GHz}$ & $7 \mathrm{GHz}$ & $8 \mathrm{GHz}$ \\
\hline SCR (dB) & 1.08 & 2.37 & 5.38 & 1.08 & 4.20 & 9.57 & 1.43 & 2.89 & 4.95 \\
\hline FWHM (mm) & 10.03 & 10.12 & 10.00 & 10.12 & 10.12 & 10.00 & 9.50 & 9.50 & 8.71 \\
\hline $\mathrm{PA}(\mathrm{mm})$ & 10.46 & 4.06 & 5.64 & 6.44 & 7.23 & 4.06 & 8.56 & 12.19 & 5.39 \\
\hline
\end{tabular}

Bifocusing algorithm. The color map represents the reconstructed normalized frequency averaged electric contrast at the surface of the phantom. In the areas where the phantom is homogeneous, i.e. in the background simulating colon tissue, the contrast is negligible and the corresponding image pixels have low values. Contrarily, if the contrast is high, i.e. in the polyp location, the image pixels present a maximum value. By comparing the images at the different frequencies, we can observe that the polyp is clearly detected in all the images but with different amounts of artifacts. $8 \mathrm{GHz}$ is the frequency that provides the most robust and clean reconstructions.

To quantify the imaging quality, we use three different metrics [27]. First, the signal to clutter ratio (SCR) compares the maximum target response to the maximum clutter (no target) response. The second and third metrics evaluate how accurate is the target positioning. The full width half maximum (FWHM) measures the distance between the peak response of the reconstructed target and the point where its response has dropped to the half. It is expressed in $\mathrm{mm}$ and gives an idea of the algorithm accuracy in retrieving the target size. Finally, the position accuracy (PA), measures the distance in mm between the peak response of the reconstructed target and the center of the real target. Table III shows the metrics evaluated for the previous phantoms' reconstructions. As anticipated in Fig. 15, the image obtained at $8 \mathrm{GHz}$ presents less clutter (i.e. highest SCR value). The best spatial resolution, quantified by FWHM and PA, is obtained for higher frequencies. The FWHM provides a good estimate of the radius of the bubble when the size of the polyp is greater than the resolution. Very accurate results are obtained even with flat or smaller protruded polyps.

\section{CONCLUSIONS}

The paper presents the design, fabrication and evaluation a proof-of-concept microwave applicator for colonoscopy. The applicator is composed by one transmitting and one receiving cavity backed U-shaped slot antennas fed by L-shaped microstrip line. The antenna elements are compact and present a good matching (return loss $-22 \mathrm{~dB}$ ) and isolation (cross coupling -25 and $-30 \mathrm{~dB}$ for the transmission-reception and side by side configurations respectively) at $8 \mathrm{GHz}$. The antenna performance is assessed with simulations and experimentally with a phantom composed by different liquids. We have reported an excellent accuracy in detecting $20 \mathrm{~mm}$-diameter flat and $10 \mathrm{~mm}$-diameter protruded lesions. This study is the first step towards the development of a fully integrated multimodal (optical and microwave) colon imaging platform that has the potential of making a positive impact on the management of CRC patients.

\section{REFERENCES}

[1] D.M. Parkin, D. Forman, F. Bray, et al. (2013). GLOBOCAN 2012 v1.0 Cancer Incidence and Mortality Worldwide. IARC, Lyon, France. [Online]. Available: http://globocan.iarc.fr

[2] M. Arnold, M. S. Sierra, M. Laversanne, I. Soerjomataram, A. Jemal, F. Bray. (2016). Global patterns and trends in colorectal cancer incidence and mortality. Gut. [Online]. 0, pp. 1-9. Available: http://gut.bmj.com/content/66/4/683

[3] R. L. Siegel, K. D. Miller, S. A. Fedewa, D. J. Ahnen, R. G. S. Meester, A. Barzi, and A. Jemal, "Colorectal cancer statistics, 2017," A Cancer Journal for Clinicians, vol. 67, pp. 177-193

[4] J. S. Lin, M. A. Piper, L A. Perdue, et al. (2016). Screening for Colorectal Cancer: A Systematic Review for the U.S. Preventive Services Task Force. Evidence Synthesis no. 135. Agency for Healthcare Research and Quality, Rockville, MD. [Online]. Available: https://jamanetwork.com/journals/jama/fullarticle/2529492

[5] A. D. Müller, A. Sonnenberg, "Prevention of colorectal cancer by flexible endoscopy and polypectomy. A case-control study of 32,702 veterans," Ann Intern Med, vol. 123, no. 12, pp. 904-910, 1995

[6] T. Lee, C. Rees, R. Blanks, et al., "Colonoscopic factors associated with adenoma detection in a national colorectal cancer screening program," Endoscopy, vol. 46, no. 3, pp. 203-211, 2014

[7] J. C. van Rijn, J. B. Reitsma, J. Stoker, P. M. Bossuyt, S. J van Deventer, E. Dekker, "Polyp miss rate determined by tandem colonoscopy: a systematic review," Am J Gastroenterol., vol. 101, no. 2, pp. 343-350, 2006

[8] N. J. Samadder, K. Curtin, T. M. Tuohy, et al., "Characteristics of missed or interval colorectal cancer and patient survival: a population-based study," Gastroenterology, vol. 146, no. 4, pp. 950-960, 2014

[9] C. P. Pox, "Controversies in Colorectal Cancer Screening," Digestion, vol. 89, pp. 274-281, 2014

[10] A. Rastogi. (2015), Clinical Review: How to Recognize Subtle Lesions in the Colon. ASGE Lead Edge. [Online]. 5(1). Available: https://www.asge.org

[11] V. Konda, S. S. Chauhan, B. K. Abu Dayyeh, et al., "Endoscopes and devices to improve colon polyp detection,". Gastrointest Endosc. vol. 81, pp. 1122-1129, 2015

[12] A. M. Buchner, "The Role of Chromoendoscopy in Evaluating Colorectal Dysplasia," Gastroenterol Hepatol, vol. 13, no. 6, pp. 336-347, 2017.

[13] J. Bernal, F. J. Sánchez, C. R. de Miguel, G. Fernández-Esparrach. (2015). Building up the Future of Colonoscopy - A Synergy between Clinicians and Computer Scientists in Screening for Colorectal Cancer with Colonoscopy. InTech. [Online]. Available: https://www.intechopen.com/

[14] S. B. Mads, Z. Wei, L. Kan, et al., "Characterizing Variability of In Vivo Raman Spectroscopic Properties of Different Anatomical Sites of Normal Colorectal Tissue towards Cancer Diagnosis at Colonoscopy," Anal Chem, vol. 87, no. 2, pp. 960-966, 2015

[15] M. Guardiola, M. Ceresa, J. Romeu, G. Fernandez-Esparrach, M. A. Gonzalez Ballester, O. Camara, "Microwave endoscopy for colorectal cancer prevention, “Int J CARS, vol. 12(Suppl 1), pp. S21, 2017

[16] M. Guardiola, S. Buitrago, G. Fernandez-Esparrach, J. M. O'Callaghan, J. Romeu, M. Cuatrecasas, H. Cordova, M. A. González-Ballester, O. Camara, "Dielectric Properties of Colon Polyps, Caner and Normal Mucosa: Ex vivo Measurements from 0.5 to $20 \mathrm{GHz}$," Med Phys, vol. 45, no. 8, pp. 3768-3782, 2018.

[17] N. Nikolova, "Introduction to Microwave Imaging," in EuMa High Frequency Technologies Series, Cambridge: Cambridge University Presss, 2017 
[18] R. Chandra, H. Zhou, I. Balasingham, S. Member, R. M. Narayanan, "On the Opportunities and Challenges in Microwave Medical Sensing and Imaging," IEEE Trans Biomed Eng, vol. 62, no. 7, pp. 1667-1682, 2015

[19] A. Winter, J. Laing, R. Paglione, F. Sterzer, "Microwave hyperthermia for brain tumors," Neurosurgery, vol. 17, no. 3, pp. 387-399, 1895

[20] M. Zakrzewski, H.Raittinen, J. Vanhala, "Comparison of Center Estimation Algorithms for Heart and Respiration Monitoring With Microwave Doppler Radar," IEEE Sens J., vol. 12, no. 3, pp. 627-634, 2012

[21] P. M. Meaney, D. Goodwin, A. H. Golnabi, et al., "Clinical Microwave Tomographic Imaging of the Calcaneus: A First-in-Human Case Study of Two Subjects," IEEE Trans Biomed Eng., vol. 59, no. 12, pp. 3304-3313, 2012

[22] S. Y. Semenov, V. G. Posukh, A. E. Bulyshev, et al., "Microwave Tomographic Imaging of the Heart in Intact Swine," J Electromagn Waves Appl., vol. 207, no. 7, pp. 873-890, 2006

[23] A. W. Preece, I. Craddock, M. Shere, L. Jones, H. L. Winton, "MARIA M4: clinical evaluation of a prototype ultrawideband radar scanner for breast cancer detection," J Med Imaging, vol. 3, no. 3, pp. 33502, 2016

[24] M. Persson, A. Fhager, H. D. Trefna, et al., "Microwave-Based Stroke Diagnosis Making Global Prehospital Thrombolytic Treatment Possible," IEEE Trans Biomed Eng., vol. 1, no. 11, pp. 2806-2817, 2014

[25] E. C. Fear, X. Li, S. C. Hagness and M. A. Stuchly, "Confocal microwave imaging for breast cancer detection: localization of tumors in three dimensions," IEEE Trans Biomed Eng., vol. 49, no. 8, pp. 812-822, 2002.

[26] L. Jofre et al., "UWB Tomographic Radar Imaging of Penetrable and Impenetrable Objects," in Proceedings of the IEEE, vol. 97, no. 2, pp. 451464, 2009.

[27] R. C. Conceicao, R. M. Capote, B. L. Oliveira, P. Almeida, M. Glavin, E. Jones, and M. O'Halloran, 'Novel multimodal PEM-UWB approach for breast cancer detection: Initial study for tumour detection and consequent classifcation," in Antennas and Propagation (EuCAP), 2013 7th European Conference on, 2013, pp. 630-634.

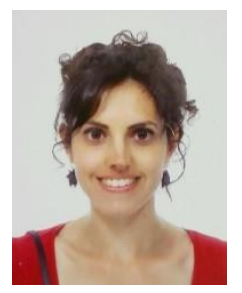

Marta Guardiola was born in Besalú, Spain, in 1984. She received the B. S. and M. S. degrees in Telecommunication Engineering, and the $\mathrm{PhD}$ in Signal Theory and Communications from Universitat Politècnica de Catalunya, Barcelona, Spain, in 2008, 2009 and 2013, respectively.

From 2006 to 2013, she was a Research Assistant with the AntennaLab research group, Universitat Politecnica de Catalunya, Barcelona. Since 2014, she has been a Visiting Professor and investigator with the BCN MedTech group, Universitat Pompeu Fabra, Barcelona. She is the author of 24 journal and conference articles and holds 1 patent. Her research interests include microwave imaging, dielectric spectroscopy, computational modelling, and antennas for microwave imaging.

Dr. Guardiola was a recipient of the UPF Social Council Knowledge Transfer Award in 2016, the IEEE International Conference on Electromagnetic Near-Field Characterization and Imaging Paper Award in 2011, and the IEEE International Symposium on Antennas and Propagation Antenna Design Contest First Prize in 2010.

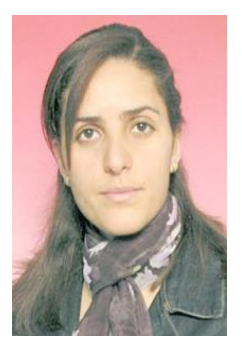

Kahina Djafri was born on January 21st, 1982, in Bejaia, Algeria. She received the Engineer degree in June 2005 from National Polytechnic School of Algers, Algeria; the M.S. degree in March 2008 from National Polytechnic School. Currently, she is Phd student in the Institute of Electrical and Electronic Engineering of University M'Hamed BOUGARA Boumerdes, Algeria.
Her research interests include Microwave propagation and Antennas.

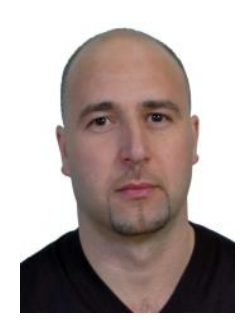

Challal Mouloud (M'09-SM'16) was born in Algiers, Algeria, in 1976. He received the Telecommunications Engineering degree in April 1999 from University of Science and Technology Houari Boumediene, BabEzzouar, Algeria, the M.S degree in December 2001 from National Polytechnic School of Algiers, Algeria, and the doctorate degree in March 2012 from University M'Hamed BOUGARA Boumerdes (UMBB), Boumerdes, Algeria.

In 2004, he joined the Department of Electrical \& Electronic Engineering, UMBB, where he was involved in teaching and as a researcher member in Telecommunications research group of Signals and Systems Laboratory. From 2007 to 2011, he was an assistant professor at UMBB. Since 2012, he has been an Associate Professor of Electrical \& Electronic Engineering at the Institute of Electrical and Electronic Engineering (IGEE, formerly called the National Institute of Electricity and Electronics - INELEC), UMBB. From 2013 to 2017, he was a responsible of master's degree program in Telecommunications at IGEE, UMBB. Since 2017, he has been a Vice-Director in charge of studies and students' related issues at IGEE, UMBB. $\mathrm{He}$ has authored/co-authored over 60 research papers in international conferences and journals. His current research interests include antennas and RF/Microwaves circuits' analysis, design, and measurement.

Dr. Challal is a founding member of the creation of the "IEEE Algeria Sub-section" where he held the post, volunteer, treasurer from 2012 to 2014 . He is also among active elements in the formation of the "IEEE Algeria Section", approved by IEEE in November 2015. In addition, he is a counselor of IEEE Student Branch of University of Boumerdes since October 2014 (date of approval). Furthermore, he is a member European Microwave Association (EuMA) since 2008 and reviewer for several refereed international journals and conferences.

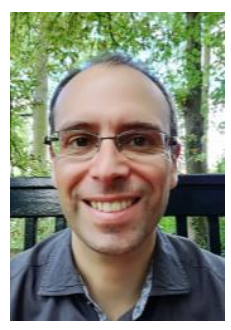

Miguel A. González Ballester holds a computer science degree from Universitat Jaume I, Spain (1996) and a doctoral degree from the University of Oxford, UK (2000).

He was awarded the prestigious Toshiba Research Fellowship and worked for two years Toshiba Medical Systems in Japan, where he developed novel, patented systems for MRI parallel imaging. In late 2001 he obtained a faculty position at INRIA (Sophia Antipolis, France), where he led research projects on medical image analysis and mathematical modelling. In 2004 he joined the University of Bern (Switzerland), as head of the medical image analysis group, and later became head of the surgical technology division at the Faculty of Medicine. From 2008 until September 2013 he was in charge of the Research Department of the company Alma IT Systems in Barcelona (Spain). In October 2013 he was awarded an ICREA Senior Research Professorship and joined the Department of Information and Communication Technologies at Universitat Pompeu Fabra in 
Barcelona, where he leads the Barcelona Center for New Medical Technologies (BCN Medtech).

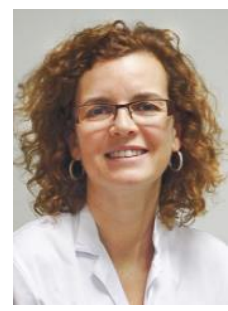

Gloria Fernandez-Esparrach was born in Barcelona, Catalunya, in 1964. She received the $\mathrm{PhD}$ degree in Medicine in 1997 for the University of Barcelona. She works at Hospital Clinic in Barcelona and her main research is on experimental Endoscopy and Endoscopic Ultrasonography. She is currently an associate professor at the University of Barcelona. She has published more than 130 original papers.

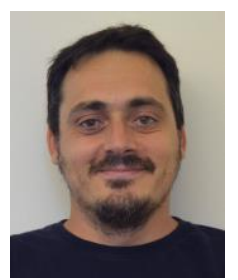

Oscar Camara received the degree in telecommunications engineering from the Universitat Politècnica de Catalunya in 1999, and the master's and Ph.D. degrees in image processing from the École Nationale Supérieure des Télécommunications, Paris, in 2000 and 2003, respectively. From 2004 to 2007, he held a Post-Doctoral position with Kings College London and University College London. In 2007, he joined Universitat Pompeu Fabra (UPF) as a Ramón y Cajal Fellow and later became an Associate Professor in 2012. He is coordinating the PhySense Research Group, which he founded in 2011. He is also one of the founders of the BCN-Medtech unit. His research is focused on methodologies at the crossroads of computational imaging and modeling areas that can be effectively used in a clinical environment, including neurology, cardiology and oncology applications.

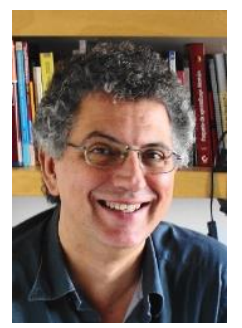

Jordi Romeu (F'12) was born in Barcelona, Spain, in 1962. He received the Ingeniero de Telecomunicación and Doctor Ingeniero de Telecomunicacion degree from the Universitat Politècnica de Catalunya (UPC), Barcelona, Spain, in 1986 and 1991, respectively.

He has been with the Electromagnetic and Photonic Engineering Group, Signal Theory and Communications Department, UPC, since 1985, where he is currently a full Professor, where he is involved in the research of antenna near-field measurements, antenna diagnostics, and antenna design. He joined the Antenna Laboratory, University of California, Los Angeles, CA, USA, in 1999, as a Visiting Scholar, under the North Atlantic Treaty Organization Scientific Program Scholarship. In 2004, he joined the University of California, Irvine, CA, USA. He has authored 50 refereed papers in international journals and 50 conference proceedings and holds several patents.

Dr. Romeu received the Grand Winner of the European IT Prize by the European Commission, for his contributions in the development of fractal antennas in 1998. 